\title{
Enumeration of Algebraic Tangles with Applications to Theta- curves and Handcuff Graphs
}

Dedicated to Professor Akio Kawauchi for his 60th birthday.

\author{
Hiromasa MORIUCHI \\ Department of Mathematics, Osaka City University, Sugimoto, Sumiyoshi-ku, Os- \\ aka 558-8585, Japan \\ e-mail: moriuchi@sci.osaka-cu.ac.jp
}

Abstract. We enumerate all algebraic tangles of seven crossings or less up to equivalence. These tangles are mutually distinguished by the corresponding links and their double. The result will be used for enumerating $\theta$-curves and handcuff graphs in a forthcoming paper.

\section{Introduction}

In [4] J. H. Conway introduced the concept of a tangle in order to enumerate knots and links. A tangle is a disjoint union of two arcs and some or no loops properly embedded in a 3-ball $B^{3}$. Two tangles $T$ and $S$ are isotopic if there is an isotopy of the 3 -ball $B^{3}$ that takes one tangle to the other while fixing each point of the boundary, and freely equivalent if there is a homeomorphism of $B^{3}$ which takes $T$ to $S$ without restriction that the endpoints stay fixed. In [14] Y. Nakanishi listed a table of algebraic tangles of five crossings or less up to isotopy by using Conway's method. In [17] H. Yamano gave a table of prime 2-string tangles of seven crossings or less up to free equivalence by using Conway's method. In [7] T. Kanenobu, H. Saito and S. Satoh classified 2-string tangles of seven crossings or less up to free equivalence by using disk-graphs. In this paper, we classify algebraic tangles of seven crossings or less up to equivalence, which is weaker than isotopy, but stronger than free equivalence (Definition 2.1). In a forthcoming paper ([11] and [12]), we give an enumeration of prime $\theta$-curves and handcuff graphs with up to seven crossings by using the result of this paper and $\theta$-polyhedra. A $\theta$-polyhedron is a connected graph embedded in 2-sphere, whose two vertices are 3 -valent, and the others are 4 -valent. We can obtain a $\theta$-curve or handcuff graph diagram from a $\theta$-polyhedron by substituting algebraic tangles for their 4 -valent vertices. This paper is organized as follows: In Section 2, we give some definitions. In Section 3 , we list a table of algebraic tangles. In Section 4 , we give some applications to $\theta$-curves and handcuff graphs.

Received August 22, 2005 and, in revised form, November 4, 2005. 2000 Mathematics Subject Classification: 57M25, 57M15.

Key words and phrases: tangle, $\theta$-curve, handcuff graph. 


\section{Algebraic tangles}

We review Conway's method [4]. We define a tangle as a pair $\left(B^{3}, t\right)$, where $t$ is a 1-manifold properly embedded in a unit 3-ball $B^{3}=\left\{(x, y, z) \in R^{3} \mid x^{2}+y^{2}+z^{2} \leq\right.$ 1 \} with four boundary components

$$
\begin{aligned}
& \mathrm{NE}=(1 / \sqrt{2}, 1 / \sqrt{2}, 0), \quad \mathrm{SE}=(1 / \sqrt{2},-1 / \sqrt{2}, 0), \\
& \mathrm{SW}=(-1 / \sqrt{2},-1 / \sqrt{2}, 0), \quad \mathrm{NW}=(-1 / \sqrt{2}, 1 / \sqrt{2}, 0) ;
\end{aligned}
$$

see Figure 1.

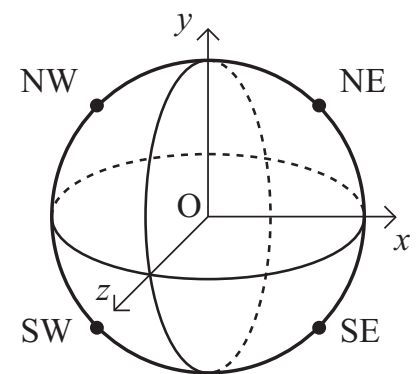

Figure 1: A 3-ball and its 4 boundary components.

Let $T=\left(B^{3}, t\right)$ be a tangle such that $t$ consists of two arcs and $n$ circles. We say $T$ is a $V^{n}$-tangle (resp. an $H^{n}$-tangle, an $X^{n}$-tangle) if $T$ has an arc connecting NE and SE (resp. NW, SW).

We present a tangle by a regular diagram as in Figure 2(a), where we use the projection $(x, y, z) \mapsto(x, y)$. Let $R$ be a tangle. We denote by $\mu R, \nu R, \rho_{x} R, \rho_{y} R, \rho_{z} R$ the tangles obtained from $R$ by reflecting with regard to the $x y$-plane; $\mu(x, y, z)=$ $(x, y,-z)$, by turning it counter-clockwise by $\pi / 2 ; \nu(x, y, z)=(-y, x, z)$, by rotating it through angle $\pi ; \rho_{x}(x, y, z)=(x,-y,-z), \rho_{y}(x, y, z)=(-x, y,-z)$, and $\rho_{z}(x, y, z)=(-x,-y, z)$, respectively. We present these tangles diagrammatically as shown in Figure 2. We call $\mu R$ the mirror image of $R$.

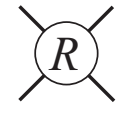

(a)

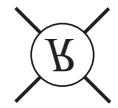

(d)

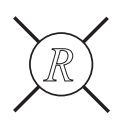

(b)

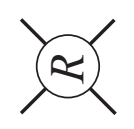

(c)

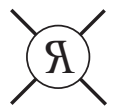

(e)

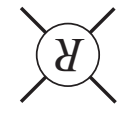

(f)

Figure 2: (a) A tangle $R$.

(b) The tangle $\mu R$.

(c) The tangle $\nu R$.

(d) The tangle $\rho_{x} R$.

(e) The tangle $\rho_{y} R$.

(f) The tangle $\rho_{z} R$. 
We say that two tangles are isotopic if there is an isotopy of the 3 -ball $B^{3}$ that takes one tangle to the other while fixing each point of the boundary, that is, their diagrams are related by a finite sequence of Reidemeister moves as shown in Figure 3 inside the circle defining the tangle while the endpoints of the strings remain fixed.

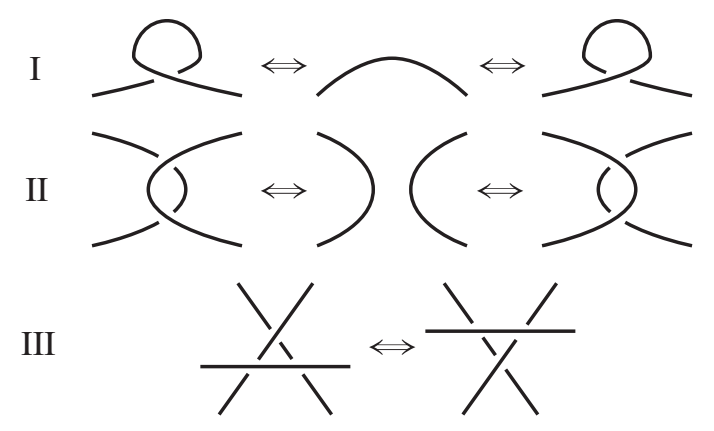

Figure 3: Reidemeister moves.

Definition 2.1. We say that two tangles $T$ and $T^{\prime}$ are equivalent if $T$ is isotopic to one of the following eight tangles:

$$
T^{\prime}, \rho_{x} T^{\prime}, \rho_{y} T^{\prime}, \rho_{z} T^{\prime}, \nu T^{\prime}, \nu \rho_{x} T^{\prime}, \nu \rho_{y} T^{\prime}, \nu \rho_{z} T^{\prime}
$$

For a tangle diagram $D$, we denote by $c(D)$ the number of crossings of $D$. The crossing number of a tangle $T$, denoted by $c(T)$, is the minimal number of $c(D)$ 's for all the diagrams $D$ which present the equivalence class of $T$.

Given two tangles $T$ and $S$, we define new tangles $T+S, T S, T+$ and $T-$ as shown in Figure 4; $T+S$ and $T S$ are the sum and product of $T$ and $S$ respectively. Notice that $T S=\rho_{x} \mu \nu(T)+S$, where $\rho_{x} \mu \nu(T)$ is the tangle obtained from $T$ by reflecting across the NW and SE diagonal line.

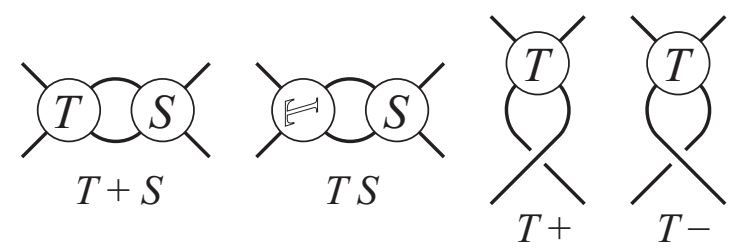

Figure 4: The operations.

The simplest tangles are the 0 and $\infty$ tangles as shown in Figures 5 (a) and (b). Further, for a positive integer $n$ we define the $n$ tangle and the $-n$ tangle as shown in Figures 5 (c) and (d), which are called integral tangles. 


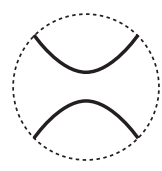

(a)

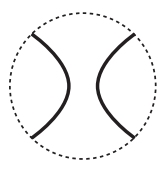

(b)

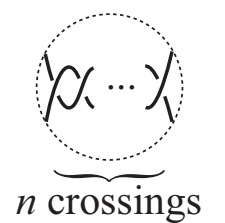

(c)

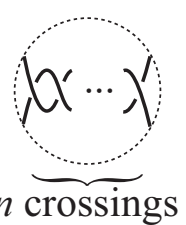

(d)

Figure 5: (a) The 0 tangle. (b) The $\infty$ tangle. (c) The $n$ tangle. (d) The $-n$ tangle.

A tangle $T$ is said to be algebraic if $T$ is obtained from the 0 and $\infty$ tangles by a finite sequence of the operations given in Figure 4. Thus, an algebraic tangle is obtained from the $0, \infty$, and integral tangles by the operations of addition and multiplication. We denote the $n$ tangle simply by $n$, and the $-n$ tangle by $\bar{n}$. For integral tangles $a_{1}, a_{2}, a_{3}, \cdots, a_{i-1}, a_{i}$, the tangle $a_{1} a_{2} a_{3} \cdots a_{i-1} a_{i}$, abbreviating $\left(\left(\cdots\left(a_{1} a_{2}\right) a_{3} \cdots a_{i-1}\right) a_{i}\right)$, is called a rational tangle. Two rational tangles $a_{1} a_{2} \cdots a_{i-1} a_{i}$ and $b_{1} b_{2} \cdots b_{j-1} b_{j}$ are isotopic if and only if the corresponding rational numbers (including $1 / 0=\infty$ )

$$
a_{i}+\frac{1}{a_{i-1}+\frac{1}{\ddots+\frac{1}{a_{2}+\frac{1}{a_{1}}}}} \text { and } \quad b_{j}+\frac{1}{b_{j-1}+\frac{1}{\ddots+\frac{1}{b_{2}+\frac{1}{b_{1}}}}}
$$

are the same.

Remark 2.2. For the above continued fraction, we can assume that each $a_{m}(1 \leq$ $m \leq i$ ) has the same sign.

The comma notation $\left(a_{1}, a_{2}, \cdots, a_{i}\right)=\left(a_{1} 0\right)+\left(a_{2} 0\right)+\cdots+\left(a_{i} 0\right)$ is preferred to the sum notation, but is only used with two or more terms in the bracket. Figure 6 shows the step-by-step formation of two algebraic tangles 2111 and 21,2 as examples.

A tangle $T=\left(B^{3}, t\right)$ is said to be splittable if there exists a disk $\Delta$ such that $\Delta$ does not meet $t$, but splits two $\operatorname{arcs}$ of $t$ in $B^{3}$.

Let $T$ be a tangle. We define the numerator, $N(T)$, and denominator, $D(T)$, the links as shown in Figure 7. We call the set of links $\{N(T), D(T)\}$ the corresponding links for $T$. Clearly, we have

Proposition 2.3. Suppose that $T$ and $S$ are equivalent tangles. Then their corresponding links $\{N(T), D(T)\}$ and $\{N(S), D(S)\}$ present the same set of isotopic links. 


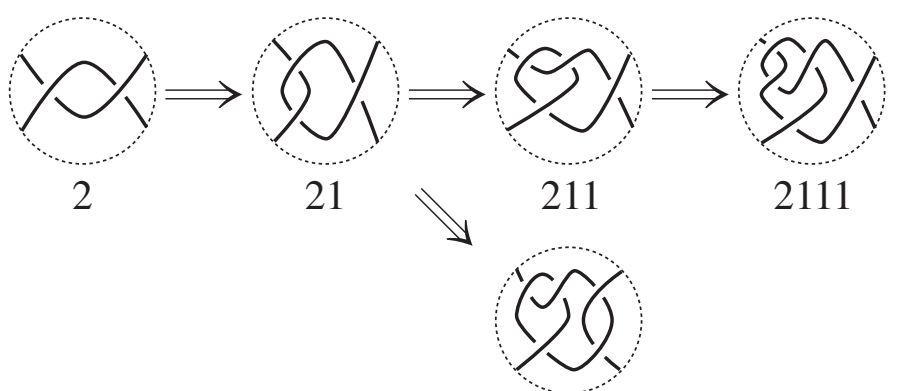

21,2

Figure 6: Algebraic tangles.

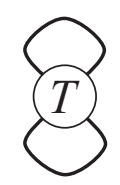

(a)

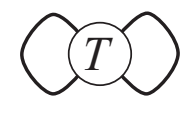

(b)

Figure 7: (a) The numerator $N(T)$. (b) The denominator $D(T)$. 8

Let $R$ be a tangle. We define the double, $W(R)$ by the link as shown in Figure

$$
W(R)=N\left(R+\rho_{y} \mu R\right) .
$$

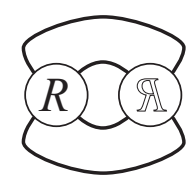

Figure 8: The double $W(R)$.

Clearly, we have

Proposition 2.4. Suppose that $T$ and $S$ are equivalent tangles. Then, their doubles $W(T)$ and $W(S)$ are isotopic.

\section{Table of algebraic tangles}

We list a table of unsplittable algebraic tangles with seven crossings or less up to equivalence in Definition 2.1. However, we list either $T$ or $\mu T$ for each tangle $T$, even if they are not equivalent. 
Theorem 3.1. Table 1 exhibit diagrams of unsplittable algebraic tangles with up to seven crossings.

Links in the second column correspond to Rolfsen's knot table [15]. Specifically, 0 is the trivial knot, $L_{1} \# L_{2}$ is a connected sum of links $L_{1}$ and $L_{2}$, and $\bar{L}$ is the mirror image of $L$. The last column gives the type of the algebraic tangle, where $X=X^{0}, H=H^{0}, V=V^{0}$.

Table 1: Algebraic tangles with up to 7 crossings.

\begin{tabular}{lcl}
$T$ & $(N(T), D(T))$ & type \\
\hline \hline 1 & $(0,0)$ & $X$ \\
\hline 2 & $\left(2_{1}^{2}, 0\right)$ & $H$ \\
\hline 3 & $\left(3_{1}, 0\right)$ & $X$ \\
21 & $\left(\overline{3_{1}}, 2_{1}^{2}\right)$ & $V$ \\
\hline 4 & $\left(4_{1}^{2}, 0\right)$ & $H$ \\
31 & $\left(4_{1}^{2}, \overline{3_{1}}\right)$ & $H$ \\
22 & $\left(4_{1}, 2_{1}^{2}\right)$ & $V$ \\
211 & $\left(4_{1}, 3_{1}\right)$ & $X$ \\
2,2 & $\left(4_{1}^{2}, 2_{1}^{2} \# 2_{1}^{2}\right)$ & $V^{1}$ \\
$2, \overline{2}$ & $\left(0_{1}^{2}, 2_{1}^{2} \# 2_{1}^{2}\right)$ & $V^{1}$ \\
\hline 5 & $\left(5_{1}, 0\right)$ & $X$ \\
41 & $\left(\overline{5_{1}}, \overline{4_{1}^{2}}\right)$ & $V$ \\
32 & $\left(5_{2}, \overline{3_{1}}\right)$ & $X$ \\
311 & $\left(\overline{5_{2}}, 4_{1}^{2}\right)$ & $V$ \\
23 & $\left(\overline{5_{2}}, 2_{1}^{2}\right)$ & $V$ \\
221 & $\left(5_{2}, 4_{1}\right)$ & $X$ \\
212 & $\left(\overline{5_{1}^{2}}, \overline{3_{1}}\right)$ & $H$ \\
2111 & $\left(\overline{5_{1}^{2}}, 4_{1}\right)$ & $H$ \\
$2,2+$ & $\left(5_{1}^{2}, 2_{1}^{2} \# 2_{1}^{2}\right)$ & $V^{1}$ \\
$(2,2) 1$ & $\left(5_{1}^{2}, 4_{1}^{2}\right)$ & $X^{1}$ \\
$(2,2) \overline{1}$ & $\left(0_{1}^{2}, 4_{1}^{2}\right)$ & $X^{1}$ \\
3,2 & $\left(\overline{5_{1}}, \overline{3_{1}} \# 2_{1}^{2}\right)$ & $V$ \\
$3, \overline{2}$ & $\left(0, \overline{3_{1}} \# 2_{1}^{2}\right)$ & $V$ \\
21,2 & $\left(5_{2}, 3_{1} \# 2_{1}^{2}\right)$ & $V$ \\
\hline
\end{tabular}


Table 1: Algebraic tangles with up to 7 crossings (continued).

\begin{tabular}{|c|c|c|}
\hline$T$ & $(N(T), D(T))$ & type \\
\hline 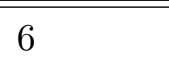 & $\left(6_{1}^{2}, 0\right)$ & $\bar{H}$ \\
\hline 51 & $\left(\overline{6_{1}^{2}}, \overline{5_{1}}\right)$ & $H$ \\
\hline 42 & $\left(6_{1}, \overline{4_{1}^{2}}\right)$ & $V$ \\
\hline 411 & $\left(\overline{6_{1}}, 5_{1}\right)$ & $X$ \\
\hline 33 & $\left(6_{2}^{2}, \overline{3_{1}}\right)$ & $H$ \\
\hline 321 & $\left(\overline{6_{2}^{2}}, \overline{5_{2}}\right)$ & $H$ \\
\hline 312 & $\left(6_{2}, 4_{1}^{2}\right)$ & $V$ \\
\hline 3111 & $\left(\overline{6_{2}}, 5_{2}\right)$ & $X$ \\
\hline 24 & $\left(\overline{6_{1}}, 2_{1}^{2}\right)$ & $V$ \\
\hline 231 & $\left(6_{1}, 5_{2}\right)$ & $X$ \\
\hline 222 & $\left(6_{3}^{2}, 4_{1}\right)$ & $H$ \\
\hline 2211 & $\left(\overline{6_{3}^{2}}, \overline{5_{2}}\right)$ & $H$ \\
\hline 213 & $\left(\overline{6_{2}}, 3_{1}\right)$ & $X$ \\
\hline 2121 & $\left(6_{2}, \overline{5_{1}^{2}}\right)$ & $V$ \\
\hline 2112 & $\left(63,4_{1}\right)$ & $X$ \\
\hline 21111 & $\left(6_{3}, 5_{1}^{2}\right)$ & $V$ \\
\hline $2,2++$ & $\left(\overline{6_{3}^{2}}, 2_{1}^{2} \# 2_{1}^{2}\right)$ & $V^{1}$ \\
\hline$(2,2) 2$ & $\left(\overline{6_{1}^{3}}, 4_{1}^{2}\right)$ & $H^{1}$ \\
\hline$(2,2) \overline{2}$ & $\left(\overline{6_{3}^{3}}, 4_{1}^{2}\right)$ & $H^{1}$ \\
\hline$(2, \overline{2}) 2$ & $\left(\overline{6_{3}^{3}}, 0_{1}^{2}\right)$ & $H^{1}$ \\
\hline $3,2+$ & $\left(6_{2}, \overline{3_{1}} \# 2_{1}^{2}\right)$ & $V$ \\
\hline $21,2+$ & $\left(6_{3}, 3_{1} \# 2_{1}^{2}\right)$ & $V$ \\
\hline$(3,2) 1$ & $\left(6_{2}, 5_{1}\right)$ & $X$ \\
\hline$(3,2) \overline{1}$ & $\left(0,5_{1}\right)$ & $X$ \\
\hline$(3, \overline{2}) \overline{1}$ & $\left(0,5_{2}\right)$ & $X$ \\
\hline$(21,2) 1$ & $\left(6_{3}, \overline{5_{2}}\right)$ & $X$ \\
\hline$(2,2+) 1$ & $\left(\overline{6_{3}^{2}}, 5_{1}^{2}\right)$ & $X^{1}$ \\
\hline$(2,2) 11$ & $\left(6_{1}^{3}, \overline{5_{1}^{2}}\right)$ & $H^{1}$ \\
\hline 4,2 & $\left(\overline{6_{1}^{2}}, \overline{4_{1}^{2}} \# 2_{1}^{2}\right)$ & $V^{1}$ \\
\hline $4, \overline{2}$ & $\left(2_{1}^{2}, \frac{1}{4_{1}^{2}} \# 2_{1}^{2}\right)$ & $V^{1}$ \\
\hline 31,2 & $\left(6_{2}^{2}, 4_{1}^{2} \# 2_{1}^{2}\right)$ & $V^{1}$ \\
\hline 22,2 & $\left(6_{1}, 4_{1} \# 2_{1}^{2}\right)$ & $V$ \\
\hline $22, \overline{2}$ & $\left(0,4_{1} \# 2_{1}^{2}\right)$ & $V$ \\
\hline
\end{tabular}


Table 1: Algebraic tangles with up to 7 crossings (continued).

\begin{tabular}{|c|c|c|}
\hline$T$ & $(N(T), D(T))$ & type \\
\hline 211,2 & $\left(\overline{6_{2}}, 4_{1} \# 2_{1}^{2}\right)$ & $V$ \\
\hline$(2,2), 2$ & $\left(\overline{6_{3}^{2}}, 4_{1}^{2} \# 2_{1}^{2}\right)$ & $V^{1}$ \\
\hline$(2,2), \overline{2}$ & $\left(\overline{4_{1}^{2}}, 4_{1}^{2} \# 2_{1}^{2}\right)$ & $V^{1}$ \\
\hline$(2, \overline{2}), 2$ & $\left(5_{1}^{2}, 0_{1}^{2} \# 2_{1}^{2}\right)$ & $V^{1}$ \\
\hline 3,3 & $\left(\overline{6_{1}^{2}}, \overline{3_{1}} \# \overline{3_{1}}\right)$ & $H$ \\
\hline $3, \overline{3}$ & $\left(0_{1}^{2}, 3_{1} \# \overline{3_{1}}\right)$ & $H$ \\
\hline 3,21 & $\left(6_{1}, 3_{1} \# \overline{3_{1}}\right)$ & $X$ \\
\hline $3, \overline{2} \overline{1}$ & $\left(3_{1}, \overline{3_{1}} \# \overline{3_{1}}\right)$ & $X$ \\
\hline 21,21 & $\left(\overline{6_{3}^{2}}, 3_{1} \# 3_{1}\right)$ & $H$ \\
\hline $2,2,2$ & $\left(6_{1}^{3}, 2_{1}^{2} \# 2_{1}^{2} \# 2_{1}^{2}\right)$ & $V^{2}$ \\
\hline $2,2, \overline{2}$ & $\left(6_{3}^{3}, 2_{1}^{2} \# 2_{1}^{2} \# 2_{1}^{2}\right)$ & $V^{2}$ \\
\hline 7 & $\left(7_{1}, 0\right)$ & $X$ \\
\hline 61 & $\left(\overline{7_{1}}, \overline{6_{1}^{2}}\right)$ & $V$ \\
\hline 52 & $\left(7_{2}, \overline{5_{1}}\right)$ & $X$ \\
\hline 511 & $\left(\overline{7_{2}}, 6_{1}^{2}\right)$ & $V$ \\
\hline 43 & $\left(7_{3}, 4_{1}^{2}\right)$ & $V$ \\
\hline 421 & $\left(\overline{7_{3}}, \overline{6_{1}}\right)$ & $X$ \\
\hline 412 & $\left(7_{1}^{2}, 5_{1}\right)$ & $H$ \\
\hline 4111 & $\left(\overline{7_{1}^{2}}, 6_{1}\right)$ & $H$ \\
\hline 34 & $\left(\overline{7_{3}}, \overline{3_{1}}\right)$ & $X$ \\
\hline 331 & $\left(7_{3}, 6_{2}^{2}\right)$ & $V$ \\
\hline 322 & $\left(7_{5}, \overline{5_{2}}\right)$ & $X$ \\
\hline 3211 & $\left(\overline{7_{5}}, 6_{2}^{2}\right)$ & $V$ \\
\hline 313 & $\left(7_{4}, 4_{1}^{2}\right)$ & $V$ \\
\hline 3121 & $\left(\overline{7_{4}}, \overline{6_{2}}\right)$ & $X$ \\
\hline 3112 & $\left(7_{2}^{2}, 5_{2}\right)$ & $H$ \\
\hline 31111 & $\left(\overline{7_{2}^{2}}, 6_{2}\right)$ & $H$ \\
\hline 25 & $\left(\overline{7_{2}}, 2_{1}^{2}\right)$ & $V$ \\
\hline 241 & $\left(7_{2}, 6_{1}\right)$ & $X$ \\
\hline 232 & $\left(7_{3}^{2}, 5_{2}\right)$ & $H$ \\
\hline 2311 & $\left(\overline{7_{3}^{2}}, \overline{6_{1}}\right)$ & $H$ \\
\hline 223 & $\left(\overline{7_{5}}, 4_{1}\right)$ & $X$ \\
\hline 2221 & $\left(7_{5}, \overline{6_{3}^{2}}\right)$ & $V$ \\
\hline
\end{tabular}


Table 1: Algebraic tangles with up to 7 crossings (continued).

\begin{tabular}{|c|c|c|}
\hline$T$ & $(N(T), D(T))$ & type \\
\hline 2212 & $\left(7_{6}, \overline{5_{2}}\right)$ & $X$ \\
\hline 22111 & $\left(\overline{7_{6}}, \overline{6_{3}^{2}}\right)$ & $V$ \\
\hline 214 & $\left(\overline{7_{1}^{2}}, 3_{1}\right)$ & $H$ \\
\hline 2131 & $\left(7_{1}^{2}, 6_{2}\right)$ & $H$ \\
\hline 2122 & $\left(\overline{7_{6}}, \overline{5_{1}^{2}}\right)$ & $V$ \\
\hline 21211 & $\left(7_{6}, \overline{6_{2}}\right)$ & $X$ \\
\hline 2113 & $\left(\overline{7_{2}^{2}}, 4_{1}\right)$ & $H$ \\
\hline 21121 & $\left(7_{2}^{2}, 6_{3}\right)$ & $H$ \\
\hline 21112 & $\left(7_{7}, 5_{1}^{2}\right)$ & $V$ \\
\hline 211111 & $\left(\overline{7_{7}}, 6_{3}\right)$ & $X$ \\
\hline $2,2+++$ & $\left(\overline{7_{3}^{2}}, 2_{1}^{2} \# 2_{1}^{2}\right)$ & $V^{1}$ \\
\hline$(2,2) 3$ & $\left(\overline{7_{4}^{2}}, 4_{1}^{2}\right)$ & $X^{1}$ \\
\hline$(2,2) \overline{3}$ & $\left(\overline{7_{8}^{2}}, 4_{1}^{2}\right)$ & $X^{1}$ \\
\hline$(2, \overline{2}) 3$ & $\left(\overline{7_{7}^{2}}, 0_{1}^{2}\right)$ & $X^{1}$ \\
\hline $3,2++$ & $\left(\overline{7_{5}}, \overline{3_{1}} \# 2_{1}^{2}\right)$ & $V$ \\
\hline $21,2++$ & $\left(7_{6}, 3_{1} \# 2_{1}^{2}\right)$ & $V$ \\
\hline$(3,2) 2$ & $\left(\overline{7_{4}^{2}}, 5_{1}\right)$ & $H$ \\
\hline$(3,2) \overline{2}$ & $\left(\overline{7_{7}^{2}}, 5_{1}\right)$ & $H$ \\
\hline$(3, \overline{2}) 2$ & $\left(\frac{1}{7_{7}^{2}}, 0\right)$ & $H$ \\
\hline$(3, \overline{2}) \overline{2}$ & $\left(7_{8}^{2}, 0\right)$ & $H$ \\
\hline$(21,2) 2$ & $\left(\overline{7_{5}^{2}}, \overline{5_{2}}\right)$ & $H$ \\
\hline$(21,2) \overline{2}$ & $\left(\overline{7_{8}^{2}}, \overline{5_{2}}\right)$ & $H$ \\
\hline$(2,2+) 2$ & $\left(\overline{7_{1}^{3}}, \overline{5_{1}^{2}}\right)$ & $H^{1}$ \\
\hline$(2,2) 12$ & $\left(7_{5}^{2}, \overline{5_{1}^{2}}\right)$ & $X^{1}$ \\
\hline $4,2+$ & $\left(\overline{7_{1}^{2}}, \overline{4_{1}^{2}} \# 2_{1}^{2}\right)$ & $V^{1}$ \\
\hline $31,2+$ & $\left(\overline{7_{2}^{2}}, 4_{1}^{2} \# 2_{1}^{2}\right)$ & $V^{1}$ \\
\hline $22,2+$ & $\left(\overline{7_{6}}, 4_{1} \# 2_{1}^{2}\right)$ & $V$ \\
\hline $211,2+$ & $\left(\overline{7_{7}}, 4_{1} \# 2_{1}^{2}\right)$ & $V$ \\
\hline$(2,2), 2+$ & $\left(\overline{7_{5}^{2}}, 4_{1}^{2} \# 2_{1}^{2}\right)$ & $V^{1}$ \\
\hline $3,3+$ & $\left(\overline{7_{4}}, \overline{3_{1}} \# \overline{3_{1}}\right)$ & $X$ \\
\hline $3,21+$ & $\left(7_{2}^{2}, 3_{1} \# \overline{3_{1}}\right)$ & $H$ \\
\hline $21,21+$ & $\left(7_{7}, 3_{1} \# 3_{1}\right)$ & $X$ \\
\hline
\end{tabular}


Table 1: Algebraic tangles with up to 7 crossings (continued).

\begin{tabular}{|c|c|c|}
\hline$T$ & $(N(T), D(T))$ & type \\
\hline $2,2,2+$ & $\left(7_{1}^{3}, 2_{1}^{2} \# 2_{1}^{2} \# 2_{1}^{2}\right)$ & $V^{2}$ \\
\hline$(2,2++) 1$ & $\left(7_{3}^{2}, 6_{3}^{2}\right)$ & $X^{1}$ \\
\hline$(2,2) 21$ & $\left(7_{4}^{2}, 6_{1}^{3}\right)$ & $V^{1}$ \\
\hline$(2,2) \overline{2} \overline{1}$ & $\left(7_{8}^{2}, 6_{3}^{3}\right)$ & $V^{1}$ \\
\hline$(2, \overline{2}) 21$ & $\left(7_{7}^{2}, 6_{3}^{3}\right)$ & $V^{1}$ \\
\hline$(3,2+) 1$ & $\left(7_{5}, 6_{2}\right)$ & $X$ \\
\hline$(21,2+) 1$ & $\left(\overline{7_{6}}, 6_{3}\right)$ & $X$ \\
\hline$(3,2) 11$ & $\left(7_{4}^{2}, \overline{6_{2}}\right)$ & $H$ \\
\hline$(21,2) 11$ & $\left(7_{5}^{2}, 6_{3}\right)$ & $H$ \\
\hline$(2,2+) 11$ & $\left(7_{1}^{3}, 6_{3}^{2}\right)$ & $H^{1}$ \\
\hline$(2,2) 111$ & $\left(\overline{7_{5}^{2}}, \overline{6_{1}^{3}}\right)$ & $V^{1}$ \\
\hline$(4,2) 1$ & $\left(7_{1}^{2}, 6_{1}^{2}\right)$ & $X^{1}$ \\
\hline$(4,2) \overline{1}$ & $\left(2_{1}^{2}, 6_{1}^{2}\right)$ & $X^{1}$ \\
\hline$(4, \overline{2}) \overline{1}$ & $\left(6_{2}^{2}, 2_{1}^{2}\right)$ & $X^{1}$ \\
\hline$(31,2) 1$ & $\left(7_{2}^{2}, 6_{2}^{2}\right)$ & $X^{1}$ \\
\hline$(22,2) 1$ & $\left(7_{6}, \overline{6_{1}}\right)$ & $X$ \\
\hline$(22,2) \overline{1}$ & $\left(0, \overline{6_{1}}\right)$ & $X$ \\
\hline$(22, \overline{2}) \overline{1}$ & $\left(\overline{6_{2}}, 0\right)$ & $X$ \\
\hline$(2 \quad 11,2) 1$ & $\left(77,6_{2}\right)$ & $X$ \\
\hline$((2,2), 2) 1$ & $\left(7_{5}^{2}, 6_{3}^{2}\right)$ & $X^{1}$ \\
\hline$((2,2), 2) \overline{1}$ & $\left(4_{1}^{2}, 6_{3}^{2}\right)$ & $X^{1}$ \\
\hline$((2,2), \overline{2}) \overline{1}$ & $\left(7_{7}^{2}, 4_{1}^{2}\right)$ & $X^{1}$ \\
\hline$((2, \overline{2}), 2) 1$ & $\left(7_{8}^{2}, \overline{5_{1}^{2}}\right)$ & $X^{1}$ \\
\hline$((2, \overline{2}), 2) \overline{1}$ & $\left(5_{1}^{2}, \overline{5_{1}^{2}}\right)$ & $X^{1}$ \\
\hline$(3,3) 1$ & $\left(7_{4}, 6_{1}^{2}\right)$ & $V$ \\
\hline$(3,3) \overline{1}$ & $\left(\overline{3_{1}}, 6_{1}^{2}\right)$ & $V$ \\
\hline$(3, \overline{3}) 1$ & $\left(\overline{6_{1}}, 0_{1}^{2}\right)$ & $V$ \\
\hline$(3,21) 1$ & $\left(7_{2}^{2}, \overline{6_{1}}\right)$ & $H$ \\
\hline$(3, \overline{2} \overline{1}) \overline{1}$ & $\left(6_{3}^{2}, \overline{3_{1}}\right)$ & $H$ \\
\hline$(2 \quad 1,21) 1$ & $\left(\overline{7_{7}}, 6_{3}^{2}\right)$ & $V$ \\
\hline$(2,2,2) 1$ & $\left(\overline{7_{1}^{3}}, \overline{6_{1}^{3}}\right)$ & $X^{2}$ \\
\hline$(2,2,2) \overline{1}$ & $\left(\overline{6_{3}^{3}}, \overline{6_{1}^{3}}\right)$ & $X^{2}$ \\
\hline$(2,2, \overline{2}) \overline{1}$ & $\left(6_{3}^{3}, \overline{6_{3}^{3}}\right)$ & $X^{2}$ \\
\hline
\end{tabular}


Table 1: Algebraic tangles with up to 7 crossings (continued).

\begin{tabular}{lcl}
$T$ & $(N(T), D(T))$ & type \\
\hline \hline 5,2 & $\left(\overline{7_{1}}, \overline{5_{1}} \# 2_{1}^{2}\right)$ & $V$ \\
$5, \overline{2}$ & $\left(\overline{3_{1}}, \overline{5_{1}} \# 2_{1}^{2}\right)$ & $V$ \\
41,2 & $\left(\overline{7_{3}}, 5_{1} \# 2_{1}^{2}\right)$ & $V$ \\
32,2 & $\left(7_{3}, \overline{5_{2}} \# 2_{1}^{2}\right)$ & $V$ \\
$32, \overline{2}$ & $\left(0, \overline{5_{2}} \# 2_{1}^{2}\right)$ & $V$ \\
311,2 & $\left(\overline{7_{4}}, 5_{2} \# 2_{1}^{2}\right)$ & $V$ \\
23,2 & $\left(7_{2}, 5_{2} \# 2_{1}^{2}\right)$ & $V$ \\
$23, \overline{2}$ & $\left(3_{1}, 5_{2} \# 2_{1}^{2}\right)$ & $V$ \\
221,2 & $\left(\overline{7_{5}}, \overline{5_{2}} \# 2_{1}^{2}\right)$ & $V$ \\
212,2 & $\left(\overline{7_{1}^{2}}, \overline{5_{1}^{2}} \# 2_{1}^{2}\right)$ & $V^{1}$ \\
$212, \overline{2}$ & $\left(2_{1}^{2}, \overline{5_{1}^{2}} \# 2_{1}^{2}\right)$ & $V^{1}$ \\
2111,2 & $\left(7_{2}^{2}, 5_{1}^{2} \# 2_{1}^{2}\right)$ & $V^{1}$ \\
$(3,2), 2$ & $\left(7_{5}, 5_{1} \# 2_{1}^{2}\right)$ & $V$ \\
$(3,2), \overline{2}$ & $\left(5_{2}, 5_{1} \# 2_{1}^{2}\right)$ & $V$ \\
$(3, \overline{2}), 2$ & $\left(6_{2}, 2_{1}^{2}\right)$ & $V$ \\
$(3, \overline{2}), \overline{2}$ & $\left(6_{3}, 2_{1}^{2}\right)$ & $V$ \\
$(21,2), 2$ & $\left(7_{6}, \overline{5_{2}} \# 2_{1}^{2}\right)$ & $V$ \\
$(21,2), \overline{2}$ & $\left(\overline{5_{1}}, \overline{5_{2}} \# 2_{1}^{2}\right)$ & $V$ \\
$(2,2+), 2$ & $\left(7_{3}^{2}, 5_{1}^{2} \# 2_{1}^{2}\right)$ & $V^{1}$ \\
$(2,2+), \overline{2}$ & $\left(0_{1}^{2}, 5_{1}^{2} \# 2_{1}^{2}\right)$ & $V^{1}$ \\
$((2,2) 1), 2$ & $\left(7_{4}^{2}, \overline{5_{1}^{2}} \# 2_{1}^{2}\right)$ & $V^{1}$ \\
$((2,2) \overline{1}), \overline{2}$ & $\left(7_{8}^{2}, 0_{1}^{2} \# 2_{1}^{2}\right)$ & $V^{1}$ \\
$((2, \overline{2}) 1), 2$ & $\left(7_{7}^{2}, \overline{4_{1}^{2}} \# 2_{1}^{2}\right)$ & $V^{1}$ \\
4,3 & $\left(7_{1}, \overline{4_{1}^{2}} \# 3_{1}\right)$ & $V$ \\
$4, \overline{3}$ & $\left(0, \overline{4_{1}^{2}} \# 3_{1}\right)$ & $V$ \\
31,3 & $\left(7_{3}, 4_{1}^{2} \# \overline{3_{1}}\right)$ & $V$ \\
$31, \overline{3}$ & $\left(4_{1}, \overline{4_{1}^{2}} \# 3_{1}\right)$ & $V$ \\
22,3 & $\left(7_{2}, 4_{1} \# \overline{3}_{1}\right)$ & $X$ \\
$22, \overline{3}$ & $\left(0,4_{1} \# 3_{1}\right)$ & $X$ \\
211,3 & $\left(\overline{7_{1}^{2}}, 4_{1} \# \overline{3_{1}}\right)$ & $H$ \\
$211, \overline{3}$ & $\left(4_{1}^{2}, 4_{1} \# 3_{1}\right)$ & $H$ \\
& & \\
& &
\end{tabular}


Table 1: Algebraic tangles with up to 7 crossings (continued).

\begin{tabular}{lcl}
$T$ & $(N(T), D(T))$ & type \\
\hline \hline$(2,2), 3$ & $\left(7_{3}^{2}, 4_{1}^{2} \# \overline{3_{1}}\right)$ & $X^{1}$ \\
$(2,2), \overline{3}$ & $\left(\overline{5_{1}^{2}}, 4_{1}^{2} \# 3_{1}\right)$ & $X^{1}$ \\
$(2, \overline{2}), 3$ & $\left(6_{3}^{2}, 0_{1}^{2} \# \overline{3_{1}}\right)$ & $X^{1}$ \\
4,21 & $\left(7_{2}, \overline{4_{1}^{2}} \# 3_{1}\right)$ & $V$ \\
31,21 & $\left(7_{5}, 4_{1}^{2} \# 3_{1}\right)$ & $V$ \\
22,21 & $\left(\overline{7_{3}^{2}}, 4_{1} \# 3_{1}\right)$ & $H$ \\
211,21 & $\left(\overline{7_{6}}, 4_{1} \# 3_{1}\right)$ & $X$ \\
$(2,2), 21$ & $\left(\overline{7_{1}^{3}}, 4_{1}^{2} \# 3_{1}\right)$ & $H^{1}$ \\
$(2,2), \overline{2} \overline{1}$ & $\left(\frac{6_{3}^{3}}{3}, 4_{1}^{2} \# \overline{3_{1}}\right)$ & $H^{1}$ \\
$(2, \overline{2}), 21$ & $\left(\overline{6_{1}^{3}}, 0_{1}^{2} \# 3_{1}\right)$ & $H^{1}$ \\
$3,2,2$ & $\left(7_{4}^{2}, \overline{3_{1}} \# 2_{1}^{2} \# 2_{1}^{2}\right)$ & $V^{1}$ \\
$3,2, \overline{2}$ & $\left(7_{7}^{2}, \overline{3_{1}} \# 2_{1}^{2} \# 2_{1}^{2}\right)$ & $V^{1}$ \\
$3, \overline{2}, \overline{2}$ & $\left(\overline{7_{8}^{2}}, \overline{3_{1}} \# 2_{1}^{2} \# 2_{1}^{2}\right)$ & $V^{1}$ \\
$21,2,2$ & $\left(7_{5}^{2}, 3_{1} \# 2_{1}^{2} \# 2_{1}^{2}\right)$ & $V^{1}$
\end{tabular}

Sketch of proof of Theorem 3.1. First, we enumerate the rational tangles with up to seven crossings. Second, we give algebraic (but not rational) tangle diagrams with up to seven crossings by using operations in Figure 4. Third, we classify the tangles up to equivalence.

In order to enumerate all the rational tangles of $n$ crossings, by Definition 2.1 and Remark 2.2, we produce the sequences of positive integers $a_{1} a_{2} \cdots a_{i}$ satisfying

$$
a_{1}+a_{2}+\cdots+a_{i}=n .
$$

Specifically, we construct a binary tree as follows.

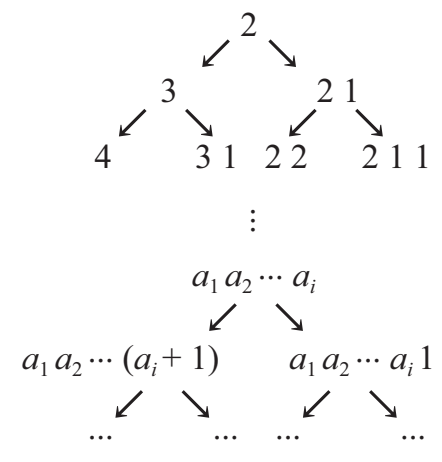


The sequence corresponds to the rational tangle $a_{1} a_{2} \cdots a_{i}$ (cf. [6]).

In order to enumerate all the algebraic (but not rational) tangle diagrams of $n$ crossings, we construct the following tree.

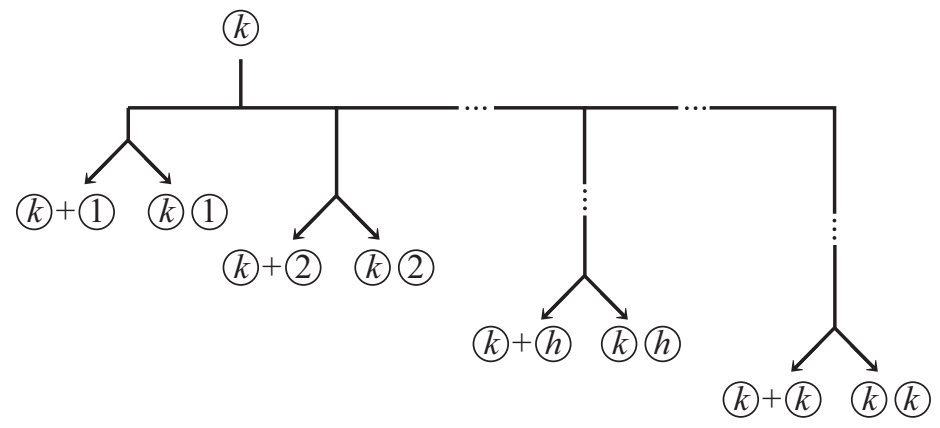

Here, (k) denotes an algebraic tangle with $k$ crossings, ( $k+(h)$ and $(k)$ (h) are new algebraic tangles with $(k+h)$ crossings (see Figure 9). By Definition 2.1, we may assume $1 \leq h \leq k$. If $(\mathbb{k})$ is the rational tangle $a_{1} a_{2} \cdots a_{i}\left(a_{m}>0\right)$, (k)+(1) corresponds to $a_{1} a_{2} \cdots\left(a_{i}+1\right)$ and (k) (1) corresponds to $a_{1} a_{2} \cdots a_{i} 1$.

Example. We give the case where $k=h=2$ in Figure 9. Note that the algebraic tangles with two crossings are $2, \overline{2}, 20, \overline{2} 0$.

Then, for each tangle $T$, we investigate the corresponding links $\{N(T), D(T)\}$ and compare them. Except for the tangles 5 and $(3,2) \overline{1}$, we show these tangles are mutually distinct by the corresponding links. In fact, $\{N(5), D(5)\}=$ $\{N((3,2) \overline{1}), D((3,2) \overline{1})\}=\left\{0,5_{1}\right\}$. However, their doubles $W(5)$ and $W((3,2) \overline{1})$ are not isotopic. So, the tangles 5 and $(3,2) \overline{1}$ are not equivalent.

\section{Applications to spatial graphs}

A spatial graph is a graph in $S^{3}$. Specifically, a $\theta$-curve $\Theta$ is a spatial graph which consists of two vertices $\left(v_{1}, v_{2}\right)$ and three edges $\left(e_{1}, e_{2}, e_{3}\right)$, such that each edge joins the vertices. A handcuff graph $\Phi$ is also a spatial graph which consists of two vertices $\left(v_{1}, v_{2}\right)$ and three edges $\left(e_{1}, e_{2}, e_{3}\right)$, where $e_{3}$ has distinct endpoints $v_{1}$ and $v_{2}$, and $e_{1}$ and $e_{2}$ are loops based at $v_{1}$ and $v_{2}$, respectively. A constituent knot is a subgraph of $\Theta$ that consists of two vertices $\left(v_{1}, v_{2}\right)$ and two edges $\left(e_{i}, e_{j}\right)$. The set of constituent knots is an invariant of $\theta$-curves. A constituent link is a subgraph of $\Phi$ that consists of two vertices $\left(v_{1}, v_{2}\right)$ and two edges $\left(e_{i}, e_{j}\right)$. The constituent link is an invariant of handcuff graphs.

From Theorem 3.1, we can enumerate special $\theta$-curves and handcuff graphs. For a tangle $T$, we define a spatial graph diagram $G(T)$ as shown in Figure 10 (cf. [10]). If $T$ is a $V^{0}$-tangle or an $X^{0}$-tangle, then $G(T)$ is a $\theta$-curve. And if $T$ is an $H^{0}$-tangle, then $G(T)$ is a handcuff graph. 


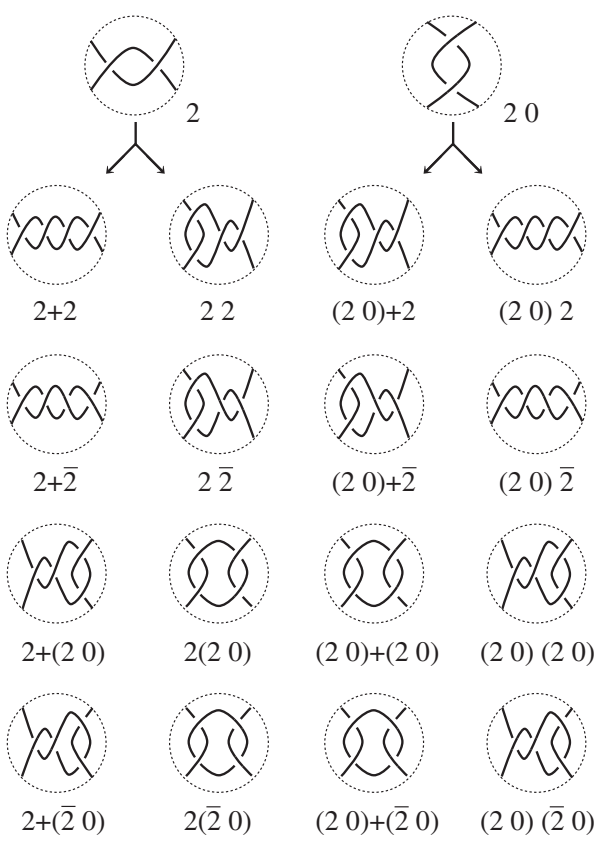

Figure 9: The case $k=h=2$.

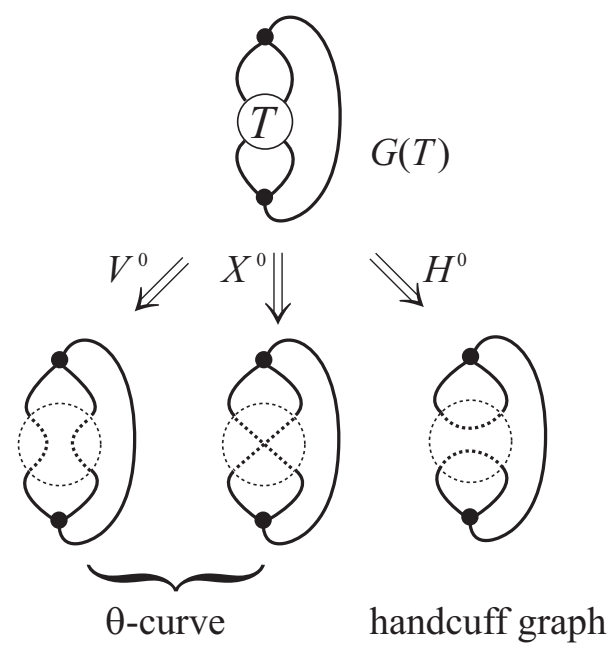

Figure 10: $G(T)$ produces a $\theta$-curve or a handcuff graph.

We give an enumeration of special $\theta$-curves with up to seven crossings as in Table 2. Knots in the second column correspond to Rolfsen's knot table [15], and 
$\theta$-curves in the last column correspond to Litherland's table [9]. The $\theta$-curves are ordered so that their constituent knots are in lexicographic order. A knot $\bar{K}$ and a $\theta$-curve $\bar{\Theta}$ denote the mirror images of $K$ and $\Theta$, respectively.

Example. For the tangle $3, \overline{2}$, we obtain the $\theta$-curve by $G(T)$. Its constituent knots are $\overline{3_{1}}$ and two trivial knot. By deformation as in Figure 11, we conclude the $\theta$-curve is $3_{1}$.

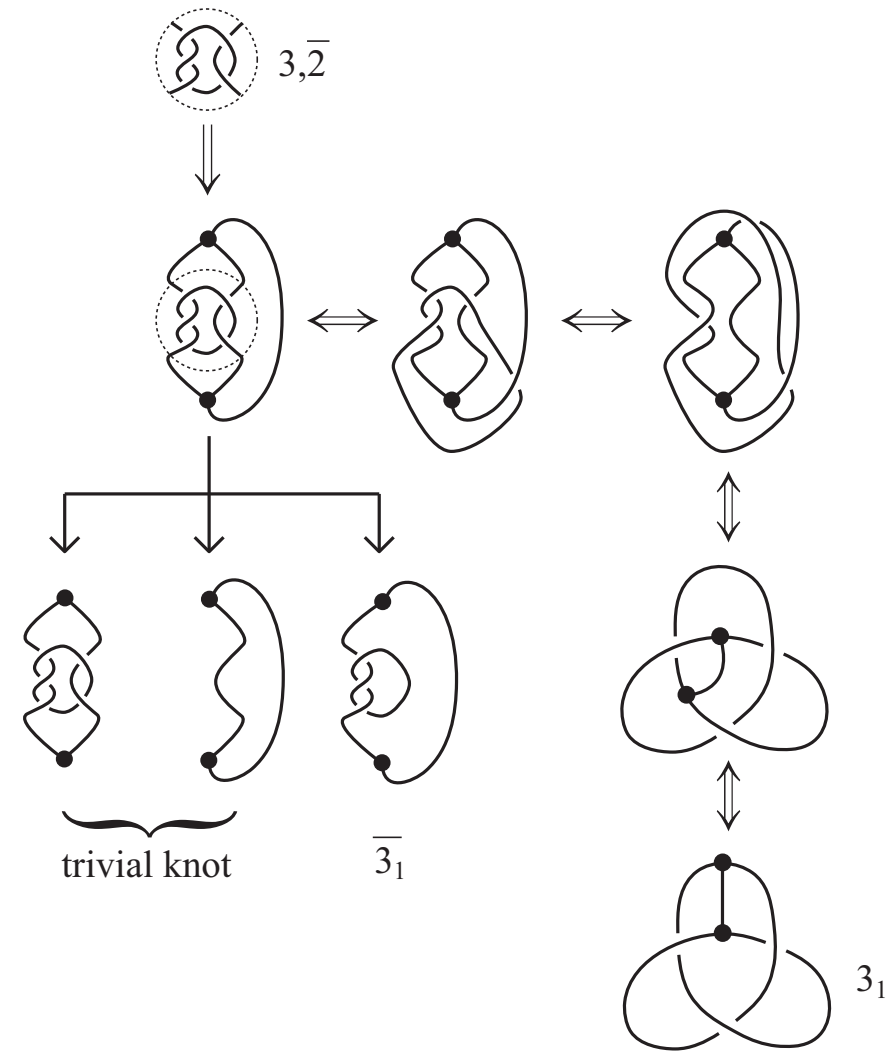

Figure 11: The tangle $3, \overline{2}$ produces the $\theta$-curve 3 . 
Table 2: $\theta$-curves with up to 7 crossings.

\begin{tabular}{|c|c|c|}
\hline Tangle & constituent knot & $\Theta$ \\
\hline 1 & $0,0,0$ & trivial \\
\hline 3 & $3_{1}, 0,0$ & $\overline{3_{1}}$ \\
\hline 21 & $\overline{3_{1}}, 0,0$ & $3_{1}$ \\
\hline 22 & $4_{1}, 0,0$ & $4_{1}$ \\
\hline 211 & $4_{1}, 0,0$ & $\overline{4_{1}}$ \\
\hline 5 & $5_{1}, 0,0$ & $5_{3}$ \\
\hline 41 & $\overline{5_{1}}, 0,0$ & $\overline{5_{3}}$ \\
\hline 32 & $5_{2}, 0,0$ & $5_{6}$ \\
\hline 321 & $\overline{5_{2}}, 0,0$ & $\overline{56}$ \\
\hline 23 & $\overline{5_{2}}, 0,0$ & $\overline{55}$ \\
\hline 221 & $5_{2}, 0,0$ & $5_{5}$ \\
\hline 3,2 & $\overline{5_{1}}, \overline{3_{1}}, 0$ & $\overline{5_{4}}$ \\
\hline $3, \overline{2}$ & $\overline{3_{1}}, 0,0$ & $3_{1}$ \\
\hline 21,2 & $5_{2}, 3_{1}, 0$ & $5_{7}$ \\
\hline 42 & $66_{1}, 0,0$ & $6_{5}$ \\
\hline 411 & $\overline{6_{1}}, 0,0$ & $\overline{6_{5}}$ \\
\hline 312 & $6_{2}, 0,0$ & $6_{9}$ \\
\hline 3111 & $\overline{6_{2}}, 0,0$ & $\overline{6_{9}}$ \\
\hline 24 & $\overline{6_{1}}, 0,0$ & $\overline{6_{6}}$ \\
\hline 231 & $61,0,0$ & $6_{6}$ \\
\hline 213 & $6_{2}, 0,0$ & $6_{10}$ \\
\hline 2121 & $\overline{6_{2}}, 0,0$ & $\overline{6_{10}}$ \\
\hline 2112 & $6_{3}, 0,0$ & $\overline{6_{14}}$ \\
\hline 21111 & $63,0,0$ & $6_{14}$ \\
\hline $3,2+$ & $\overline{6_{2}}, \overline{3_{1}}, 0$ & $\overline{6_{12}}$ \\
\hline $21,2+$ & $6_{3}, 3_{1}, 0$ & $\overline{6_{16}}$ \\
\hline$(3,2) 1$ & $6_{2}, 3_{1}, 0$ & $6_{12}$ \\
\hline$(3,2) \overline{1}$ & $3_{1}, 0,0$ & $\overline{3_{1}}$ \\
\hline$(3, \overline{2}) \overline{1}$ & $5_{2}, 3_{1}, 0$ & $5_{7}$ \\
\hline$(21,2) 1$ & $6_{3}, \overline{3_{1}}, 0$ & $6_{16}$ \\
\hline 22,2 & $6_{1}, 4_{1}, 0$ & $6_{8}$ \\
\hline $22, \overline{2}$ & $4_{1}, 0,0$ & $4_{1}$ \\
\hline 211,2 & $\overline{6_{2}}, 4_{1}, 0$ & $\overline{6_{13}}$ \\
\hline 3,21 & $\overline{6_{1}}, 0,0$ & $\overline{6_{7}}$ \\
\hline $3, \overline{2} \overline{1}$ & $3_{1}, 0,0$ & $\overline{5_{2}}$ \\
\hline
\end{tabular}


Table 2: $\theta$-curves with up to 7 crossings (continued).

\begin{tabular}{|c|c|c|}
\hline Tangle & constituent knot & $\Theta$ \\
\hline 7 & $77_{1}, 0,0$ & $7_{25}$ \\
\hline 61 & $\overline{7_{1}}, 0,0$ & $\overline{7_{25}}$ \\
\hline 52 & $7_{2}, 0,0$ & $\overline{7_{29}}$ \\
\hline 511 & $\overline{7_{2}}, 0,0$ & $7_{29}$ \\
\hline 43 & $7_{3}, 0,0$ & $7_{33}$ \\
\hline 421 & $\overline{7_{3}}, 0,0$ & $\overline{7_{33}}$ \\
\hline 34 & $\overline{7_{3}}, 0,0$ & $\overline{7_{34}}$ \\
\hline 331 & $7_{3}, 0,0$ & $7_{34}$ \\
\hline 322 & $7_{5}, 0,0$ & $\overline{7_{43}}$ \\
\hline 3211 & $7_{5}, 0,0$ & $7_{43}$ \\
\hline 313 & $7_{4}, 0,0$ & $7_{38}$ \\
\hline 3121 & $\overline{7_{4}}, 0,0$ & $77_{38}$ \\
\hline 25 & $\overline{7_{2}}, 0,0$ & $7_{28}$ \\
\hline 241 & $7_{2}, 0,0$ & $\overline{7_{28}}$ \\
\hline 223 & $75,0,0$ & $\overline{7_{44}}$ \\
\hline 2221 & $\overline{7_{5}}, 0,0$ & $7_{44}$ \\
\hline 2212 & $7_{6}, 0,0$ & $\frac{x}{7_{53}}$ \\
\hline 22111 & $\overline{7_{6}}, 0,0$ & $7_{53}$ \\
\hline 2122 & $\overline{7_{6}}, 0,0$ & $7_{50}$ \\
\hline 21211 & $7_{6}, 0,0$ & $\frac{50}{7_{50}}$ \\
\hline 21112 & $7_{7}, 0,0$ & $7_{59}$ \\
\hline 211111 & $\overline{7_{7}}, 0,0$ & $\frac{.}{759}$ \\
\hline $3,2++$ & $\overline{7_{5}}, \overline{3_{1}}, 0$ & $7_{46}$ \\
\hline $21,2++$ & $7_{6}, 3_{1}, 0$ & $\overline{7_{56}}$ \\
\hline $22,2+$ & $\overline{7_{6}}, 4_{1}, 0$ & $7_{57}$ \\
\hline $211,2+$ & $\overline{7_{7}}, 4_{1}, 0$ & $\overline{7_{65}}$ \\
\hline $3,3+$ & $7_{4}, 0,0$ & $\frac{00}{739}$ \\
\hline $21,21+$ & $7_{7}, 0,0$ & $7_{62}$ \\
\hline$(3,2+) 1$ & $7_{5}, 3_{1}, 0$ & $\overline{7_{46}}$ \\
\hline$(21,2+) 1$ & $\overline{7_{6}}, \overline{3_{1}}, 0$ & $7_{56}$ \\
\hline$(22,2) 1$ & $7_{6}, 4_{1}, 0$ & $\overline{7_{57}}$ \\
\hline$(22,2) \overline{1}$ & $4_{1}, 0,0$ & $\overline{4_{1}}$ \\
\hline$(22, \overline{2}) \overline{1}$ & $\overline{6_{2}}, 4_{1}, 0$ & $\overline{6_{13}}$ \\
\hline$(2 \quad 11,2) 1$ & $7_{7}, 4_{1}, 0$ & $7_{65}$ \\
\hline
\end{tabular}


Table 2: $\theta$-curves with 7 crossings (continued).

\begin{tabular}{|c|c|c|}
\hline Tangle & constituent knot & $\Theta$ \\
\hline$(3,3) 1$ & $7_{4}, 0,0$ & $7_{39}$ \\
\hline$(3,3) \overline{1}$ & $\overline{3_{1}}, 0,0$ & $5_{2}$ \\
\hline$(3, \overline{3}) 1$ & $\overline{6_{1}}, 0,0$ & $\frac{\bar{c}}{67}$ \\
\hline$(21,21) 1$ & $\overline{7_{7}}, 0,0$ & $\overline{7_{62}}$ \\
\hline 5,2 & $\overline{7_{1}}, \overline{5_{1}}, 0$ & $\overline{7_{27}}$ \\
\hline $5, \overline{2}$ & $\overline{5_{1}}, \overline{3_{1}}, 0$ & $\frac{21}{54}$ \\
\hline 41,2 & $\overline{7_{3}}, 5_{1}, 0$ & $\overline{736}$ \\
\hline 32,2 & $7_{3}, \overline{5_{2}}, 0$ & $7_{37}$ \\
\hline $32, \overline{2}$ & $\overline{5_{2}}, 0,0$ & $\overline{55}$ \\
\hline 311,2 & $\overline{7_{4}}, 5_{2}, 0$ & $\overline{7_{42}}$ \\
\hline 23,2 & $7_{2}, 5_{2}, 0$ & $\overline{7_{32}}$ \\
\hline $23, \overline{2}$ & $5_{2}, 3_{1}, 0$ & $5_{7}$ \\
\hline 221,2 & $\overline{7_{5}}, \overline{5_{2}}, 0$ & $7_{49}$ \\
\hline$(3,2), 2$ & $7_{5}, 5_{1}, 0$ & $\overline{7_{48}}$ \\
\hline$(3,2), \overline{2}$ & $5_{1}, 5_{2}, 0$ & $7_{18}$ \\
\hline$(3, \overline{2}), 2$ & $6_{2}, 0,0$ & $6_{11}$ \\
\hline$(3, \overline{2}), \overline{2}$ & $6_{3}, 0,0$ & $6_{15}$ \\
\hline$(21,2), 2$ & $\overline{7_{6}}, \overline{5_{2}}, 0$ & $7_{58}$ \\
\hline$(21,2), \overline{2}$ & $\overline{5_{1}}, \overline{5_{2}}, 0$ & $\overline{7_{18}}$ \\
\hline 4,3 & $\overline{7_{1}}, \overline{3_{1}}, 0$ & $\overline{7_{26}}$ \\
\hline $4, \overline{3}$ & $3_{1}, 0,0$ & $\frac{20}{52}$ \\
\hline 31,3 & $7_{3}, \overline{3_{1}}, 0$ & $7_{35}$ \\
\hline $31, \overline{3}$ & $4_{1}, 3_{1}, 0$ & $66_{4}$ \\
\hline 22,3 & $7_{2}, 0,0$ & $\overline{7_{30}}$ \\
\hline $22, \overline{3}$ & $0,0,0$ & $\overline{5_{1}}$ \\
\hline 4,21 & $7_{2}, 3_{1}, 0$ & $\overline{7_{31}}$ \\
\hline 31,21 & $7_{5}, 3_{1}, 0$ & \\
\hline 211,21 & $\frac{}{7_{6}}, 0,0$ & $7_{54}$ \\
\hline
\end{tabular}

We also give an enumeration of special handcuff graphs with up to seven crossings as in Table 3. Links in the second column correspond to Rolfsen's knot table [15], and handcuff graphs in the last column correspond to our table [12]. The handcuff graphs are ordered so that their constituent links are in lexicographic order. A link $\bar{L}$ and a handcuff graph $\bar{\Phi}$ denote the mirror images of $L$ and $\Phi$, respectively. Moreover, $\#_{3}$ denotes an order 3 vertex connected sum (see [16]). 
Table 3: Handcuff graphs with up to 7 crossings.

\begin{tabular}{|c|c|c|}
\hline Tangle & constituent link & $\Phi$ \\
\hline 2 & $2_{1}^{2}$ & $22_{1}$ \\
\hline 4 & $4_{1}^{2}$ & $4_{1}$ \\
\hline 31 & $\overline{4_{1}^{2}}$ & $\overline{4_{1}}$ \\
\hline 212 & $5_{1}^{2}$ & $5_{1}$ \\
\hline 2111 & $\overline{5_{1}^{2}}$ & $\overline{5_{1}}$ \\
\hline 6 & $6_{1}^{2}$ & $6_{5}$ \\
\hline 51 & $\frac{1}{6_{1}^{2}}$ & $\overline{6_{5}}$ \\
\hline 33 & $6_{2}^{\frac{1}{2}}$ & $6_{7}$ \\
\hline 321 & $\overline{6_{2}^{2}}$ & $\overline{6_{7}}$ \\
\hline 222 & $6_{3}^{2}$ & $6_{8}$ \\
\hline 2211 & $\overline{6_{3}^{2}}$ & $\overline{6_{8}}$ \\
\hline 3,3 & $\overline{6_{1}^{2}}$ & $6_{6}$ \\
\hline $3, \overline{3}$ & $0_{1}^{\frac{1}{2}}$ & $2_{1} \#_{3} 2_{1}$ \\
\hline 21,21 & $\frac{1}{6_{3}^{2}}$ & $6_{9}$ \\
\hline 412 & $7_{1}^{2}$ & $7_{18}$ \\
\hline 4111 & $\overline{7_{1}^{2}}$ & $\overline{7_{18}}$ \\
\hline 3112 & $7_{2}^{2}$ & $7_{22}$ \\
\hline 31111 & $\overline{7_{2}^{2}}$ & $\overline{7_{22}}$ \\
\hline 232 & $\underline{7_{3}^{2}}$ & $7_{26}$ \\
\hline 2311 & $\overline{7_{3}^{2}}$ & $\overline{7_{26}}$ \\
\hline 214 & $\overline{7_{1}^{2}}$ & $\overline{7_{19}}$ \\
\hline 2131 & $\underline{7_{1}^{2}}$ & $7_{19}$ \\
\hline 2113 & $\overline{7_{2}^{2}}$ & $7_{23}$ \\
\hline 21121 & $7_{2}^{2}$ & $\overline{7_{23}}$ \\
\hline$(3,2) 2$ & $\overline{7_{4}^{2}}$ & $7_{28}$ \\
\hline$(3,2) \overline{2}$ & $\overline{7_{7}^{2}}$ & $7_{35}$ \\
\hline$(3, \overline{2}) 2$ & $\overline{7_{7}^{2}}$ & $7_{35}$ \\
\hline$(3, \overline{2}) \overline{2}$ & $\overline{7_{8}^{2}}$ & $\overline{7_{36}}$ \\
\hline$(21,2) 2$ & $\overline{7_{5}^{2}}$ & $7_{30}$ \\
\hline$(21,2) \overline{2}$ & $\overline{7_{8}^{2}}$ & $7_{36}$ \\
\hline $3,21+$ & $7_{2}^{2}$ & $\underline{7_{24}}$ \\
\hline$(3,2) 11$ & $7_{4}^{2}$ & $\overline{7_{28}}$ \\
\hline
\end{tabular}


Table 3: Handcuff graphs with up to 7 crossings (continued).

\begin{tabular}{lcl} 
Tangle & constituent link & $\Phi$ \\
\hline \hline$(21,2) 11$ & $\overline{7_{5}^{2}}$ & $\overline{7_{30}}$ \\
$(3,21) 1$ & $\overline{7_{2}^{2}}$ & $\overline{7_{24}}$ \\
$(3, \overline{2} \overline{1}) \overline{1}$ & $6_{3}^{2}$ & 69 \\
211,3 & $\overline{7_{1}^{2}}$ & $7_{20}$ \\
$211, \overline{3}$ & $4_{1}^{2}$ & $\overline{6_{4}}$ \\
22,21 & $\overline{7_{3}^{2}}$ & $7_{21}$
\end{tabular}

Acknowledgements. The author would like to thank Professor Taizo Kanenobu and Professor Akio Kawauchi for their valuable advice and encouragement.

\section{References}

[1] C. C. Adams, "The Knot Book. An elementary introduction to the mathematical theory of knots", W. H. Freeman and Company, New York, 1994.

[2] A. Caudron, Classification des nœuds et des enlacements, Publications Mathématiques d'Orsay 82, 4(1982). Université de Paris-Sud, Département de Mathématique, Orsay.

[3] T. D. Cochran, D. Ruberman, Invariants of tangles, Math. Proc. Cambridge. Philos. Soc., 105(2)(1989) 299-306.

[4] J. H. Conway, "An enumeration of knots and links, and some of their algebraic properties", Computational Problems in Abstract Algebra (Proc. Conf. Oxford, 1967), Pergamon Press(1970) 329-358.

[5] T. Kanenobu, Tangle surgeries on the double of a tangle and link polynomials, Kobe J. Math., 19(1-2)(2002), 1-19.

[6] T, Kanenobu, T. Sumi, Polynomial invariants of 2-bridge knots through 22 crossings, Math. Comp., 60(202)(1993), 771-778, S17-S28.

[7] T. Kanenobu, H. Saito, S. Satoh, Tangles with up to seven crossings, Proceedings of the Winter Workshop of Topology/Workshop of Topology and Computer (Sendai, 2002/Nara, 2001). Interdiscip. Inform. Sci., 9(1)(2003), 127-140.

[8] L. H. Kauffman, State models and the Jones polynomial, Topology, 26(3)(1987), 395407.

[9] R. A. Litherland, A table of all prime theta-curves in $S^{3}$ up to 7 crossings, a let$\operatorname{ter}(1989)$. 
[10] H. Moriuchi, An enumeration of theta-curves with up to 7 crossings (in Japanese), Master Thesis, Osaka City Univ., 2004.

[11] H. Moriuchi, An enumeration of theta-curves with up to seven crossings, submitted.

[12] H. Moriuchi, A table of handcuff graphs with up to seven crossings, OCAMI Studies 1 Knot theory for Science Objects (to appear).

[13] K. Murasugi, Knot Theory and Its Applications, Translated from the 1993 Japanese original by Bohdan Kurpita. Birkhäuser Boston, Inc., Boston, MA, 1996.

[14] Y. Nakanishi, Alexander invariants of links (in Japanese), Master Thesis, Kobe Univ., 1980.

[15] D. Rolfsen, Knots and Links, Mathematics Lecture Series, No. 7. Publish or Perish, Inc., Berkeley, Calif., 1976.

[16] K. Wolcott, The knotting of theta-curves and other graphs in $S^{3}$, Geometry and topology (Athens, Ga., 1985), Lecture Notes in Pure and Appl. Math., Dekker, New York, 105(1987), 325-346.

[17] H. Yamano, Classification of tangles of 7 crossings or less (in Japanese), Master Thesis, Tokyo Metrop. Univ., 2001. 